\section{Discussion}

Giant cell arteritis is a relatively common disease in the elderly ${ }^{1}$ and can affect almost all the large arteries, including the aorta. ${ }^{34}$ The superficial temporal and ophthalmic arteries are often affected, causing headache and visual disturbance. Polymyalgia rheumatica frequently accompanies these symptoms. The intracranial arteries are rarely affected, ${ }^{34}$ but this has been reported in some cases. ${ }^{15}$

The pathogenesis is unknown. ${ }^{34}$ Fragmentation of the internal elastic lamina, an inflammatory infiltrate of mixed mononuclear and polymorphonuclear leucocytes, and characteristic multinucleated giant cells are the histological hallmarks of the disease. ${ }^{4}$ Immunological mechanisms have been postulated but the precise pathogenic processes have not been elucidated. ${ }^{3}$ Survival rates for GCA are similar to those of an age matched population, ${ }^{1}$ and fatal complications in well treated patients are rare, even in those with chronic relapsing disease. ${ }^{2}$ Early mortality (within six weeks of presentation) ${ }^{1}$ while uncommon, is usually associated with arteritis and brain stem infarction, or coronary arteritis and myocardial infarction. ${ }^{12}$ Late mortality (more than six weeks after presentation) due to cerebral infarction is very rare. ${ }^{2}$ Death has been attributed to insufficient corticosteroid treatment. ${ }^{1}$ In fatal cases due to vertebro- basilar disease, necropsy showed either intracranial and extracranial arteritis with thrombosis $^{1}$ or extracranial vertebral arteritis, with unaffected intracranial vessels. ${ }^{1}$

Our case is the only one in which dissection of the intracranial portion of the vertebral artery due to GCA is recorded, although dissection of the aorta has been documented before. ${ }^{1}$ It is not clear whether our patient had continuous active arteritis or a relapse following reduction of her maintenance treatment. It is clear, however, that severe GCA with fatal complications occurred with a normal ESR, without recurrence of initial symptoms, and in spite of eight months of continuous treatment. This case also shows that active GCA may cause microscopic vertebral artery dissection.

1 Save-Soderbergh J, Malmvall BE, Anderson R, Bengtsson B. Giant cell arteritis as a cause of death. $¥ A M A 1986$ B. Giant cell.

2 Graham E, Holland A, Avery A, Ross Russel RW. Prognosis in giant cell arteritis. $\mathrm{Br}$ Med $\mathcal{f} 1981 ; 282$ 269-71.

3 Fauci AS, Haynes BF, Katz P. The spectrum of vasculitis; clinical, pathologic, immunologic and therapeutic considerations. Ann Intern Med 1978;89(part 1):660-76.

4 Graham DI. Vascular disorders of the central nervous system. In: Adams JH, Duchen LW, eds. Greenfield's neuropathology. 5th edn. London: Edward Arnold, 1992: ne28-9.

5 Collado A, Santamaria J, Ribalta T, Cinta Cid M, Ca-Nete JD, Tolosa E. Giant cell arteritis presenting with ipsilateral hemiplegia and lateral medullary syndrome. Eur Neurol 1989;29:266-8.

\title{
Renal failure caused by leukaemic infiltration in chronic lymphocytic leukaemia
}

\author{
J K Phillips, P S Bass, G Majumdar, D R Davies, N F Jones, T C Pearson
}

St Thomas's Hospital, Lambeth Palace Road, London SE1 7EH

Department of

Haematology

J K Phillips

G Majumdar

T C Pearson

Department of Pathology

P S Bass

D R Davies

Correspondence to: DrJ K Phillips

Accepted for publication 1 July 1993

\begin{abstract}
A case of B-CLL which was complicated by chronic renal failure due to leukaemic infiltration of the kidney is reported. Treatment with chlorambucil, prednisolone, and renal bed irradiation resulted in a substantial improvement in renal function which persisted until the the patient's death from marrow failure some eight years later. The temporal association between treatments and response suggested that renal bed radiotherapy had contributed to the improvement in renal function. This case is one of only two reported cases in which chronic renal failure due to CLL has been treated with radiotherapy. It is unique in that the renal response was shown histologically.

Leukaemic infiltration of the kidney is common in CLL but, characteristically, is not associated with renal impairment. An improvement in renal function has
\end{abstract}

been described in two patients with acute renal failure after chemotherapy.

(F Clin Pathol 1993;46:1131-1133)

\section{Case report}

A 60 year old man presented with generalised lymphadenopathy without other abnormal physical signs. Investigation showed the following: haemoglobin $152 \mathrm{~g} / \mathrm{l}$; white cell count $11.3 \times 10^{9} / 1$ (lymphocytes $6.8 \times 10^{9} / 1$ ); and platelet count $120 \times 10^{9} / 1$. The peripheral blood film showed a lymphocytosis with occasional "smudge" cells. Peripheral blood lymphocyte surface markers were consistent with B cell CLL (91\% positive for HLA-DR, $81 \%$ positive for CD19, $91 \%$ positive for CD5, $9 \%$ positive for CD2, $5 \%$ weakly positive for SmIg, $0.5 \%$ positive for FMC7, $1 \%$ positive for $\mathrm{CD} 10,3 \%$ positive for $\mathrm{CD} 25$ ). A bone marrow aspirate showed that $65 \%$ of nucleated cells were small lymphocytes. A bone 


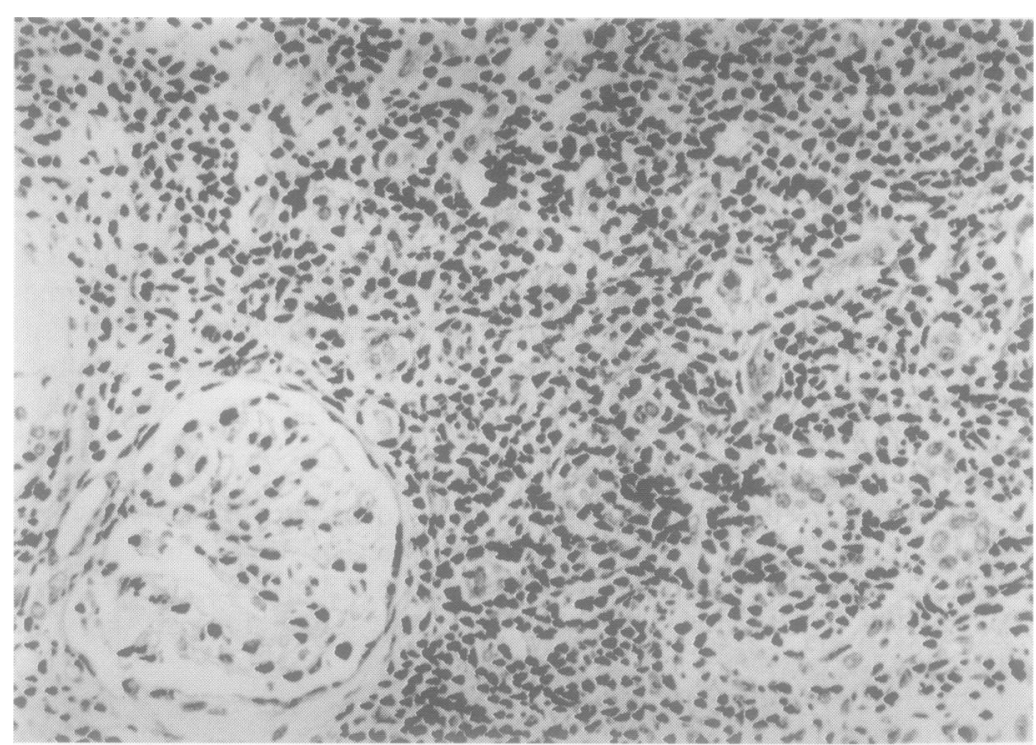

Figure 1 Renal biopsy specimen taken before radiotherapy showing a heavy lymphoid infiltrate in the interstitium, pronounced tubular atrophy, and a relatively normal glomerulus.

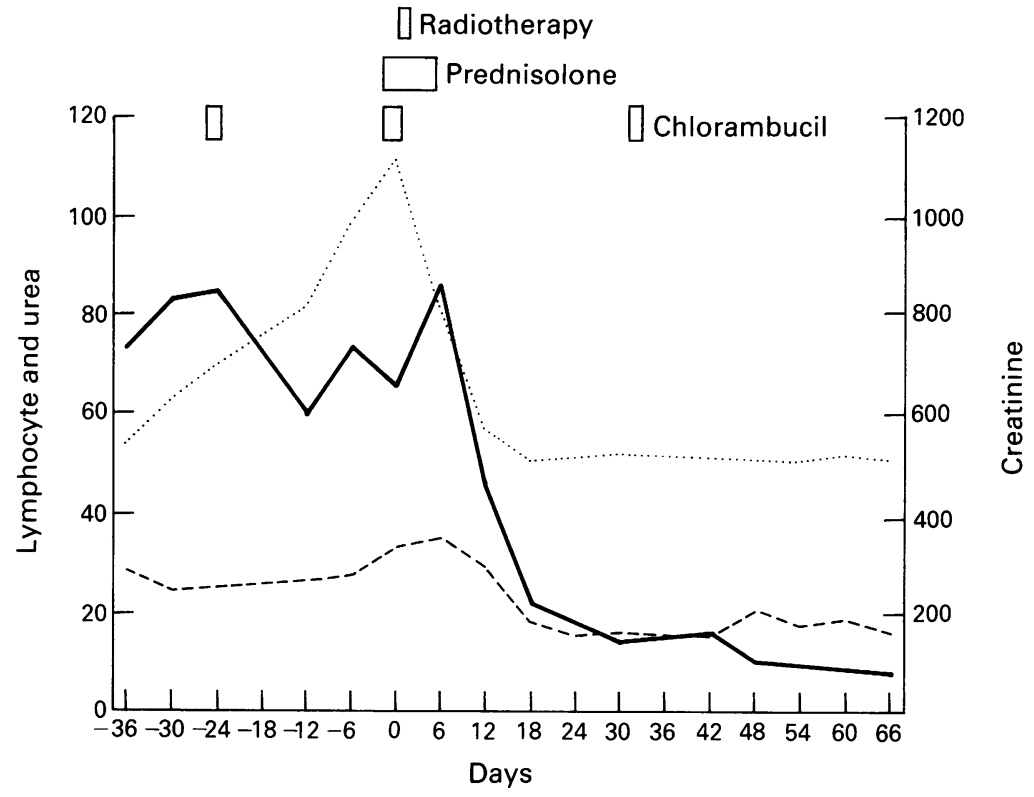

Figure 2 Temporal relations of changes in peripheral lymphocyte count and serum creatinine to radiotherapy and chemotherapy. _urea; ......... creatine; - . - lymphocyte count. the preceding three weeks. Examination showed generalised lymphadenopathy without splenomegaly, mild hypertension (blood pressure $150 / 100 \mathrm{~mm} \mathrm{Hg}$ ) and grade II hypertensive retinopathy. Investigation showed: haemoglobin $106 \mathrm{~g} / \mathrm{l}$; white cell count $90.5 \times$ $10^{9} / 1$ (lymphocytes $81.5 \times 10^{9} / 1$ ); a platelet count of $153 \times 109 / 1$; urea of $27.8 \mathrm{mmol} / \mathrm{l}$; creatinine of $563 \mu \mathrm{mol}$; creatinine clearance $15 \mathrm{ml} /$ minute; and a urinary protein excretion of $0.7 \mathrm{~g} /$ day. Serum calcium and uric acid values remained normal. Moderate glycosuria was noted on urinalysis in the presence of a plasma glucose concentration of $4.8 \mathrm{mmol} / \mathrm{l}$. A renal ultrasound scan showed normal sized kidneys with no evidence of obstructive nephropathy; the only abnormality on plain tomography was the presence of slightly irregular renal outlines. A needle renal biopsy specimen showed severe tubular atrophy with wide separation of tubules by interstitial connective tissue heavily infiltrated by small lymphocytes exhibiting the same surface marker pattern as the peripheral blood lymphocytes. The infiltrate was more pronounced in the cortex than in the medulla. Twenty nine of 33 glomeruli showed ischaemic collapse with periglomerular fibrosis, the remainder showing global sclerosis. The arteries showed moderate fibroelastic intimal proliferation and mild hyaline arteriolar sclerosis was present (fig 1). These appearances were interpreted as showing severe ischaemic nephrosclerosis with infiltration of the cortical interstitium by CLL.

The patient received oral chlorambucil 20 $\mathrm{mg}$ daily for two days with oral Allopurinol $200 \mathrm{mg}$ daily. Treatment for renal failure consisted of a low protein diet and oral bicarbonate. Atenolol $50 \mathrm{mg}$ daily was given for the hypertension. Three weeks later the lymphocyte count had fallen to $61 \times 10^{9} / 1$ but there had been a substantial decline in renal function with urea reaching $31.9 \mathrm{mmol} / 1$ and creatinine $1124 \mu \mathrm{mol} / 1$. Further oral treatment with chlorambucil $20 \mathrm{mg}$ once a day for three days and prednisolone $20 \mathrm{mg}$ three times a day for 10 days was given in addition to low dose renal bed irradiation ( $400 \mathrm{cGy}$ in two fractions given on successive days). Over the next two weeks the serum creatinine concentration fell to $496 \mu \mathrm{mol} / 1$ and the urea to $17.9 \mathrm{mmol} / \mathrm{l}$. This was followed by a fall in the lymphocyte count to $14 \times 10^{9} / 1$ at about five weeks after the second course of chemotherapy. Figure 2 shows the changes in serum creatinine and lymphocyte count in relation to treatment during this period.

Treatment for CLL was continued with monthly courses of chlorambucil for a total of nine courses. One month after completing chemotherapy the patient was asymptomatic, with lymphadenopathy confined to two small cervical nodes. The lymphocyte count was normal at $2.6 \times 10^{9} / 1$ and renal function had shown further slight improvement with a serum creatinine of $362 \mu \mathrm{mol} / 1$ and urea of $16.1 \mathrm{mmol} / \mathrm{l}$. Renal biopsy was repeated five months after radiotherapy and this showed a pronounced reduction in the extent of lymphocyte count for 12 months. He then complained of lethargy, a change in his sense of taste, and urinary frequency with frothy, smoky urine. He had lost $8 \mathrm{~kg}$ in weight over 


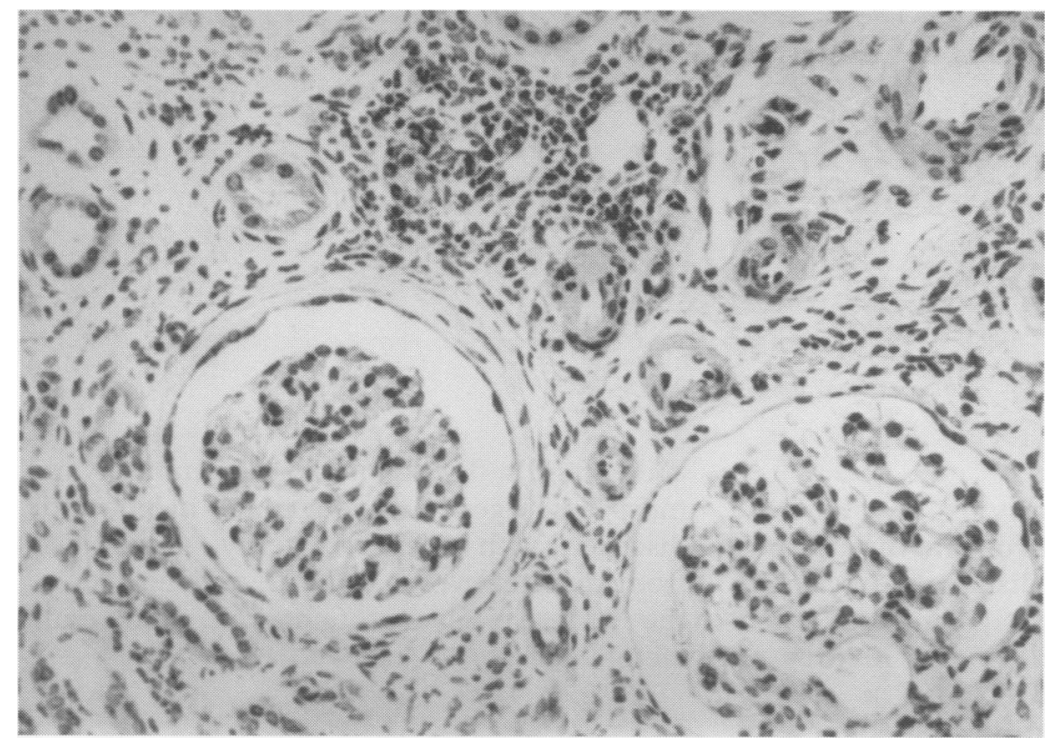

Figure 3 Renal biopsy specimen taken after radiotherapy showing a less extensive infiltrate. Tubular atrophy is still apparent and there is periglomerular fibrosis.

cortical lymphocytic infiltration, although ischaemic nephrosclerotic changes remained unchanged (fig 3).

Subsequently the patient received intermittent treatment with oral chlorambucil with or without prednisolone whenever there was evidence of disease progression. Renal function remained stable until he died, eight years later, of septicaemia secondary to bone marrow failure associated with extensive marrow infiltration by CLL.

\section{Discussion}

Necropsy studies report a prevalence of renal infiltration in CLL of between $63 \%$ and $90 \% .^{1-3}$ The histological appearance of infiltration of the kidney by CLL has been described in two previous publications. A case report describes foci of lymphocytes scattered irregularly throughout the kidney, more common in the cortex than the medulla. ${ }^{4}$ In a study of 42 cases Schwartz et al observed discrete lymphocytic foci in the cortical interstitium, particularly the subcapsular cortex, and along the vasa recta at the corticomedullary junction. ${ }^{2}$ In this study leukaemic foci were associated with fibrosis, tubular atrophy, and glomerulosclerosis.

In our patient initial treatment with chlorambucil resulted in an improvement in the peripheral lymphocyte count but was accompanied by a deterioration in renal function without evidence of renal cause for this. It was unlikely to have been due to uric acid nephropathy as the serum uric acid was never significantly raised and chemotherapy was accompanied by prophylaxis with Allopurinol. Light chain nephropathy is also unlikely as a paraprotein was not demonstrated in either plasma or urine. No evidence of amyloid was seen on the renal biopsy specimen and the serum calcium concentration remained normal. It has been suggested that the kidney may act as a sanctuary site for leukaemic cells in response to treatment with chemotherapy which may explain our finding of worsening renal failure in the face of otherwise responsive disease. ${ }^{5}$ This is consistent with the subsequent improvement in renal function accompanied by documented reduction in lymphocytic infiltration of the kidney.

The improvement in renal function followed treatment with a combination of chlorambucil, prednisolone, and renal bed irradiation. This response is not likely to be related to chlorambucil as previous exposure to this agent had been ineffective. Either prednisolone or radiotherapy might have been responsible, but it is clear from fig 2 that the renal function improved before there was any evidence of an effect on peripheral lymphocytes. A possible synergistic effect of local radiotherapy with chemotherapy cannot, however, be excluded.

Although renal infiltration by $\mathrm{CLL}$ is common, reports of impairment of renal function associated with such infiltration are rare. ${ }^{4-8}$ Acute renal failure responded to oral chlorambucil in one case ${ }^{7}$ and chronic renal failure seemed to respond to combination chemotherapy (cyclophosphamide, vincristine, and prednisolone) in another. ${ }^{6}$ Merrill and Jackson reported that renal bed irradiation (of unspecified dose) was effective in one patient, ${ }^{4}$ but Saggi et al found renal radiotherapy ( 850 rads to one and 700 rads to the other kidney over three weeks), together with oral prednisolone and chlorambucil, to be ineffective in their patient. ${ }^{8}$ In each of these four cases response was assessed on the basis of changes in renal function alone. Our patient is at present unique in that prolonged improvement in chronic renal failure followed treatment and was accompanied by a demonstrable reduction in lymphocytic infiltration of the kidneys.

Our case shows that when chronic renal failure occurs in CLL, leukaemic infiltration of the kidney should be considered as a possible cause. Renal bed irradiation may then be effective treatment, leading to improvement in renal function.

1 Kirshbaum JD, Preuss FS. Leukemia: A clinical and pathologic study of one hundred and twenty-three fatal cases in a series of 14,400 necropsies. Arch Intern Med 1943;71:777-92.

2 Schwartz JB, Samsuddin AM. The effects of leukemic infiltrates in various organs in chronic lymphocytic leukemia. Hum Pathol 1981;12:432-40.

3 Barcos M, Lane W, Gomez GA, Han T, Freeman A, Priesler H, Henderson E. An autopsy study of 1206 acute and chronic leukemias. Cancer 1987;60:827-37.

4 Merrill D, Jackson $H$. The renal complications of leukemia. N Engl f Med 1943;228:271-6.

5 Lightwood R, Barrie H, Butler N. Observations on 100 cases of leukaemia in childhood. Br Med $\mathcal{F} 1960 ; 5175$ : cases of

6 Pagniez DC, Fenaux P, Delvallez L, Dequiedt P, Gosselin B, Tacquet A. Reversible renal failure due to specific infiltration in chronic lymphocytic leukemia. $A m \mathcal{F} M e d$ 1988;85:579-80.

7 Tucker B, Brown AL, d'Ardenne AJ, Cattell WR Reversible renal failure due to renal infiltration and associated tubulointerstitial disease in chronic lymphocytic leukaemia. Nephrol Dial Transplant 1990;5:616-8.

8 Saggi S, Calandri C, Muhlfelder T, Choi H, Kahn T, Kaji D. Renal failure due to leukaemic infiltration in chronic lymphocytic leukaemia. Nephrol Dial Transplant 1990;5: 1051-2. 